\title{
Molecule-assisted ferromagnetic atomic chain formation
}

\author{
Manohar Kumar, Kiran Kumar Vidya Sethu, and Jan M. van Ruitenbeek ${ }^{\dagger}$ \\ Huygens-Kamerlingh Onnes Laboratory, Leiden University, Niels Bohrweg 2, 2333 CA Leiden, The Netherlands
}

(Received 2 November 2014; revised manuscript received 18 April 2015; published 4 June 2015)

\begin{abstract}
One dimensional systems strongly enhance the quantum character of electron transport. Such systems can be realized in $5 d$ transition metals $\mathrm{Au}, \mathrm{Pt}$, and $\mathrm{Ir}$, in the form of suspended monatomic chains between bulk leads. Atomic chains between ferromagnetic leads would open up many perspectives in the context of spin-dependent transport and spintronics, but the evidence suggests that for pure metals only the mentioned three $5 d$ metals are susceptible to chain formation. It has been argued that the stability of atomic chains made up from ferromagnetic metals is compromised by the same exchange interaction that produces the local moments. Here we demonstrate that magnetic atomic chains can be induced to form in break junctions under the influence of light molecules. Explicitly, we find deuterium assisted chain formation in the $3 d$ ferromagnetic transition metals $\mathrm{Fe}$ and $\mathrm{Ni}$. Chain lengths up to eight atoms are formed upon stretching the ferromagnetic atomic contact in deuterium atmosphere at cryogenic temperatures. From differential conductance spectra vibronic states of $\mathrm{D}_{2}$ can be identified, confirming the presence of deuterium in the atomic chains. Shot noise spectroscopy indicates the presence of weakly spin polarized transmission channels.
\end{abstract}

PACS number(s): 73.63.Rt, 85.75.-d, 72.25.-b, 72.70.+m

\section{INTRODUCTION}

The spontaneous formation of chains of metal atoms was observed experimentally for $\mathrm{Au}$ atomic contact breaking cycles by two groups simultaneously in 1998 [1,2]. Since then, atomic chain formation for other transition metal elements, in pure form or assisted by small molecules or impurities, has been reported by various groups [3-12]. The experiments were mainly performed using the mechanically controllable break junction (MCBJ) technique, or using scanning tunneling microscope (STM) methods. In a typical break junction experiment, such as reported by Yanson et al., a wire of the metal of interest, prepared with a weak link in its midpoint, is stretched in a controlled fashion using a piezoelectric element, while the conductance of the sample is recorded simultaneously. During the breaking of the wire the weak link is reduced to atomic size just before it breaks. The controlled stretching in the last stages of the atomic contact can lead to pulling new atoms into a chain arrangement, depending on the type of metal under study. The process of atomic chain formation requires the breaking force $F_{0}$ to be large. This force equals the maximum string tension in the atomic chain at the point of inflection of the total energy as a function of stretching. Further, the difference in cohesion energy of atom in the leads $\left(E_{\text {Lead }}\right)$ and the chain $\left(E_{\text {Chain }}\right)$ needs to be small enough $\delta E=E_{\text {Chain }}-E_{\text {Lead }}$. When an atomic contact is elongated, the energy of the system increases to the point where an atom from the lead can overcome the energy required to join the chain. Upon addition of this new atom in the chain, the interatomic bond length relaxes, thus lowering the mechanical strain energy of the system. Apart from intrinsic atomic chain formation for pure systems, chain formation induced by small molecules was reported by Thijssen et al. [7]. Depending on the type of metal, the presence of gases such as $\mathrm{D}_{2}, \mathrm{H}_{2}, \mathrm{O}_{2}$, and

\footnotetext{
"Present address: Low temperature Laboratory, Aalto University, Puumiehenkuja 2B Otaniemi, Espoo, Finland.

†Corresponding author: ruitenbeek@ physics.leidenuniv.nl
}

$\mathrm{N}_{2}$ may lead to a linear metal-molecule bond that is stronger than the pure linear metal-metal bond, which increases in the break force $\left(F_{0}\right)$. This was proposed to explain the observations by Thijssen et al. of very long chain formation in $\mathrm{Ag}$ and $\mathrm{Cu}$ break junctions in the presence of $\mathrm{O}_{2}$ molecules. Here, we report a similar approach to investigate chain formation in the ferromagnetic atomic contacts for $\mathrm{Fe}$ and $\mathrm{Ni}$ in the presence of deuterium, $\mathrm{D}_{2}$. Under clean conditions of cryogenic vacuum, ferromagnetic atomic contacts for $\mathrm{Fe}, \mathrm{Ni}$, and $\mathrm{Co}$ fail to form the atomic chains of more than two atoms long [13-15]. In contrast, in the presence of $\mathrm{D}_{2}$ ferromagnetic atomic contacts readily form stable and long atomic chains. Note that the use of $\mathrm{D}_{2}$ instead of $\mathrm{H}_{2}$ is for practical purposes only. Indeed, similar effects of $\mathrm{H}_{2}$ induced formation of short atomic chains have recently been reported for $\mathrm{Co}$ and $\mathrm{Pd}[16,17]$. The ferromagnetic atomic contacts have many transmission channels taking part in conductance. The presence of $\mathrm{D}_{2}$ in the ferromagnetic atomic contacts limits the number of eigenchannels to two, and shot noise measurements indicate that these are weakly polarized spin channels.

\section{A. Experimental procedure}

$\mathrm{Ni}$ and $\mathrm{Fe}$ atomic contacts are formed from metallic wires of purity $99.998 \%$ by breaking under cryogenic vacuum, at liquid helium temperatures. We use the mechanically controllable break junction technique, similar to that used by Thijssen et al. [7]. The contact breaking process, induced by stretching the metallic wire at the weak spot, can be viewed as the formation of a neck that gradually becomes narrower. In the last stages of stretching an atomic size contact is formed, just before breaking. Stretching of the contact with sub- $\AA$ precision is facilitated by the use of a piezoelement. We start by characterizing the native ferromagnetic atomic junctions by collecting conductance breaking traces into a histogram of conductance values, and verifying the expected characteristics of atomic ferromagnetic junctions by by conductance and noise spectroscopy. 
After characterization of the atomic contacts, deuterium gas $\left(D_{2}, 99.999 \%\right)$ is introduced from a vapor source at room temperature through a capillary tube leading to the contact. This procedure requires three steps in order to reduce contamination of the atomic contacts with unspecified molecules. First, all the tubes connected at the top of the dipstick, which is sealed by a needle valve, are flushed with $\mathrm{D}_{2}$ gas. Next, the tubes are pumped back to base pressure (about $10^{-6}$ mbar). The capillary tube, running from the top of the dipstick to the cryogenic break junction stage at its bottom, ends in a small detachable nozzle with an opening facing towards the sample. The capillary tube and nozzle are baked out at $150{ }^{\circ} \mathrm{C}$ for a day before cooling down the dipstick to liquid He temperature. By cooling to liquid helium temperatures residual gases condense to the walls, creating a very good (cryogenic) vacuum. $\mathrm{D}_{2}$ is introduced into the capillary by opening the needle valve, while keeping the nozzle at its base temperature (about $5 \mathrm{~K}$ ). During the process of introducing the gas the junction is continuously cycled between broken and closed states and conductance traces are continuously monitored. As soon as a molecule is caught in the atomic contact the atomic signature of the contact is suppressed and the molecular signature is seen in the conductance traces: for $\mathrm{D}_{2}$, steps appear in the conductance traces near the quantum unit of conductance, $1 \mathrm{G}_{0}=2 e^{2} / h$, resulting in a peak at $1 \mathrm{G}_{0}$ in the conductance histogram [18]. As soon as this molecular signature is seen the needle valve is closed and the tubes connected to it are pumped down to the base pressure $\left(10^{-6}\right.$ mbar). All conductance measurements for this characterization procedure are two-point dc measurements at a bias of $50 \mathrm{mV}$. For differential conductance measurements of individual stable configurations a lock-in amplifier is used with a modulation signal of amplitude $2 \mathrm{mV}$ and frequency $2.777 \mathrm{kHz}$, while sweeping dc bias voltage from $-80 \mathrm{mV}$ to $80 \mathrm{mV}$ [19]. The measurements of shot noise are done using the two-channel cross-spectrum measurement up to $100 \mathrm{kHz}$, as described in Refs. [19-22].

\section{B. Experimental observations}

Ferromagnetic junctions are gently broken in the cryogenic environment and the conductance is recorded during the breaking process. The last plateau of the breaking trace signifies a junction cross section corresponding to a single atom. This last plateau is of our interest. In the cases of $\mathrm{Ni}$ and $\mathrm{Fe}$ ferromagnetic atomic contacts the last plateaus in the conductance traces are seen near $1.3 \mathrm{G}_{0}$ and $2.1 \mathrm{G}_{0}$, respectively. The ferromagnetic atomic contacts tend to form short plateaus only, corresponding at best to a short chain of only two atoms connecting the leads at either side. Typical conductance traces and conductance histograms for $\mathrm{Fe}$ and $\mathrm{Ni}$ atomic contacts are shown in Figs. 1(a) and 1(b). Upon addition of $\mathrm{D}_{2}$, the last conductance plateau is shifted to values near $1 \mathrm{G}_{0}$. Two examples of conductance traces for $\mathrm{Ni}-\mathrm{D}_{2}-\mathrm{Ni}$ are shown in the inset of Fig. 1(c), plotted against displacement, a length scale transformed from piezovoltage (that controls the displacement of the two wire ends with respect to each other) using a calibration procedure as described in Refs. [23,24]. A conductance histogram built from thousands of such traces is shown in the main panel of Fig. 1(c). The conductance
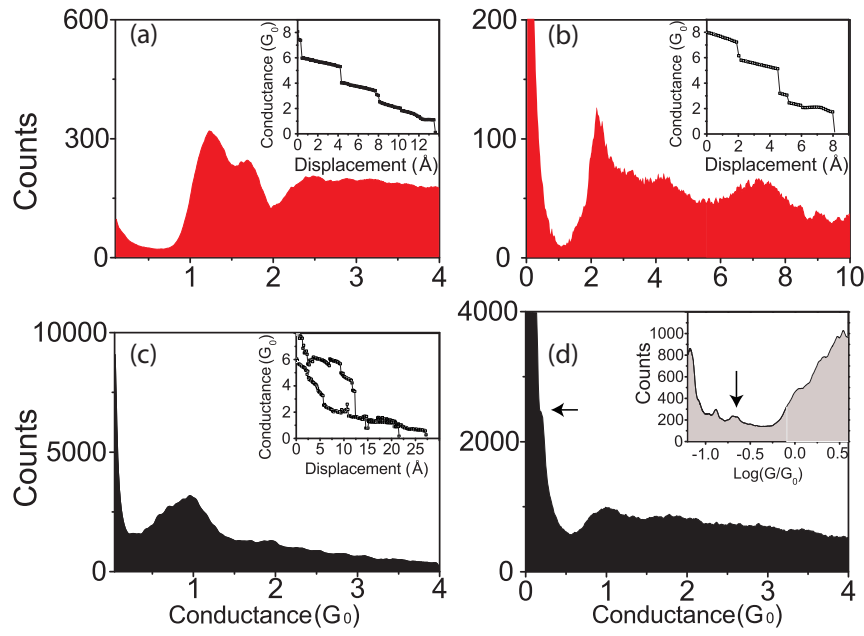

FIG. 1. (Color online) (a) Conductance histogram for Ni atomic contacts obtained from 1000 conductance traces, showing peaks at $1.3 \mathrm{G}_{0}$ and $1.6 \mathrm{G}_{0}$. The inset shows a typical conductance trace. (b) Conductance histogram of $\mathrm{Fe}$ atomic contacts obtained from 1000 conductance traces, showing peak at $2 \cdot 1 \mathrm{G}_{0}$. A typical conductance trace for Fe atomic contacts, showing a plateau at $2.1 \mathrm{G}_{0}$, is shown in inset. Its conductance monotonically decreases upon stretching of the contact and eventually gives way to breaking of the contact into the tunneling regime. Conductance histogram for $\mathrm{Ni}-\mathrm{D}_{2}-\mathrm{Ni}$ atomic contacts (c), constructed by collecting measured conductance points from 10000 conductance breaking traces. The inset shows two typical traces used in making this histogram. The conductance histogram for $\mathrm{Fe}-\mathrm{D}_{2}-\mathrm{Fe}$ atomic contacts in (d) is obtained from 5000 conductance traces. The inset shows a logarithmic conductance histogram made from the same traces, which shows the presence of a small peak at $0.25 \mathrm{G}_{0}$.

histogram shows a broad peak at $1 \mathrm{G}_{0}$ with a decaying tail at higher conductance and a small shoulder peak at $2 \mathrm{G}_{0}$. The strong peaks at $1.2 \mathrm{G}_{0}$ and $1.6 \mathrm{G}_{0}$ seen for pure $\mathrm{Ni}$ atomic contacts are completely suppressed in the presence of $\mathrm{D}_{2}$. The conductance trace shows that the length of the last plateau for this system is limited to about three atoms.

In similar experiments for $\mathrm{Fe}-\mathrm{D}_{2}-\mathrm{Fe}$, the conductance histogram shows a broad peak at $1 \mathrm{G}_{0}$ with a small shoulder peak at $0.25 \mathrm{G}_{0}$, as illustrated in Fig. $1(\mathrm{~d})$. The latter feature is enhanced in the logarithmic conductance histogram shown in the inset. In contrast to the $\mathrm{Ni}-\mathrm{D}_{2}-\mathrm{Ni}$ system $\mathrm{Fe}-\mathrm{D}_{2}-\mathrm{Fe}$ shows very long plateaus at lower conductances. Typical traces are shown in the inset of Fig. 2. The Fe- $\mathrm{D}_{2}-\mathrm{Fe}$ system shows chain lengths of five to six atoms long quite frequently, and some traces indicate up to eight atom long chains. The distribution of the lengths of the last plateaus in the breaking traces are collected in the form of length histograms. These are obtained from the lengths of the last plateaus in all traces between suitably chosen start and stop values for the conductance. In order to capture the last plateau we chose start and stop values of $1.5 \mathrm{G}_{0}$ and $0.5 \mathrm{G}_{0}$, respectively, based on the position and the width of the peak near $1 \mathrm{G}_{0}$ in the conductance histogram. We have verified that the resulting histograms do not sensitively depend on this choice. Such length histograms for $\mathrm{Ni}-\mathrm{D}_{2}-\mathrm{Ni}$ junctions do not display any distinct signature of very long chains. On the other hand, the length histogram of $\mathrm{Fe}-\mathrm{D}_{2}-\mathrm{Fe}$ given in Fig. 2 shows multiple peaks with a spacing between 


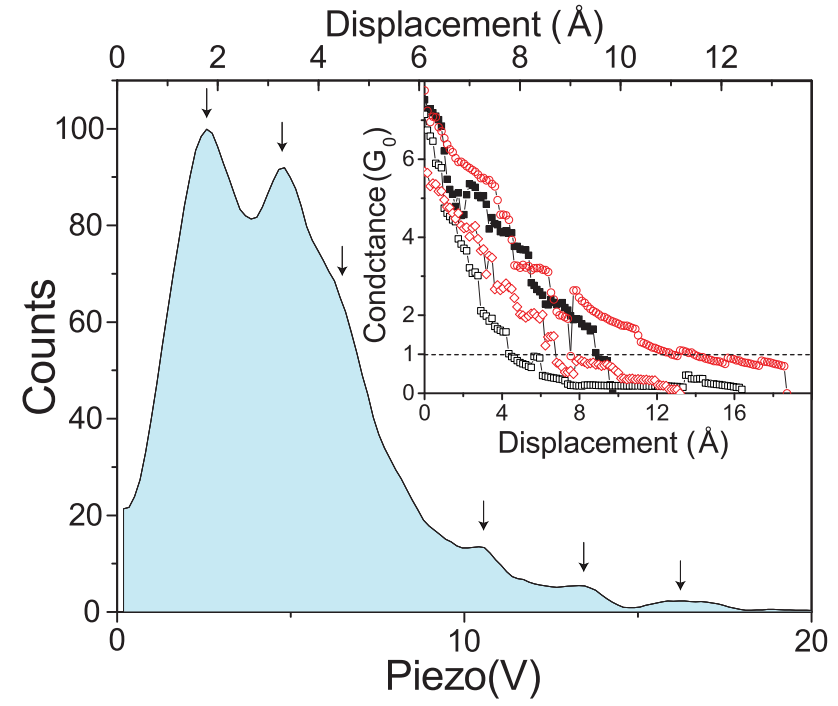

FIG. 2. (Color online) Length histogram for $\mathrm{Fe}-\mathrm{D}_{2}-\mathrm{Fe}$ atomic contacts obtained from conductance traces, showing multiple peaks with first interpeak distance of $1.63 \AA$. Peaks are marked with arrow (the third peak is shadowed by shoulder of first two peaks). The length histogram was recorded in the conductance window of $1.5 \mathrm{G}_{0}$ and $0.5 \mathrm{G}_{0}$. The inset shows typical conductance traces for $\mathrm{Fe}-\mathrm{D}_{2}-\mathrm{Fe}$. The black trace shows the presence of four steps of $1.37 \AA$ in the relevant window, which indicates the formation of a five atom long chain. The other traces show the presence of extended structures at very low conductance of $0.2-0.3 \mathrm{G}_{0}$, which are not included in this length histogram because they fall outside the selection window. The length axis of this length histogram is shown in units of voltage on the piezoelement. The appropriate proportionality constant is $1.45 \mathrm{~V} / \AA$, using the known interpeak distance in length histograms for $\mathrm{Au}$ atomic contacts for calibration $[23,24]$.

the first two prominent peaks of about $1.63 \AA$, which indicates the formation of atomic chains of distinct atomic length.

The first five peaks can loosely be interpreted as counts for two, three, four, five, and six atom long chains. The diminishing tail shows the presence of even longer chains, albeit with lower counts. The interpeak distance of $1.63 \AA$ is close to the experimental value obtained by Thijssen et al. [7] and the value calculated by Bahn et al. [25] for the $\mathrm{Au}-\mathrm{H}_{2}-\mathrm{Au}$.

We proceeded by investigating our molecule assisted ferromagnetic junction by the voltage dependence of the differential conductance (point contact spectroscopy) and shot noise measurements. The differential conductance is expected to show steps at the energies for vibration mode excitation in the junction. The sign of the step depends on the transmission probability of the conductance channels [26-29]. The differential conductance measurements for the pure ferromagnetic atomic contacts $(\mathrm{Fe}$ and $\mathrm{Ni}$ ) are dominated by the presence of a strong zero-bias anomaly that has been attributed to local Kondo scattering [15]. Typical traces for $\mathrm{Ni}_{-} \mathrm{D}_{2}-\mathrm{Ni}$ and $\mathrm{Fe}-\mathrm{D}_{2}-\mathrm{Fe}$ are shown in the Supplemental Material [30]. The differential conductance shows various features: apart from the regular step-down or step-up features anomalous peaks or dips are seen, sometimes along with zero-bias anomalies. These anomalous spikes occur at the same energies as the usual vibronic energy steps and have been interpreted in terms of

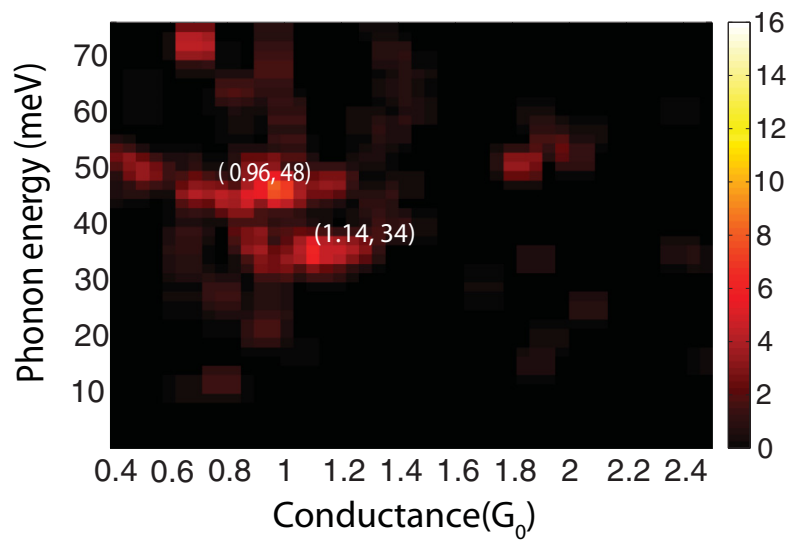

FIG. 3. (Color online) Density plot of the energies of the vibrationally induced two level systems (VITLS) for Fe- $\mathrm{D}_{2}-\mathrm{Fe}$ ferromagnetic molecular junctions (obtained from the differential conductance for the positive bias) plotted against the zero-bias conductance of the junction.

vibrationally induced two level systems (VITLS) in molecular junctions [31]. VITLS signals are usually stronger than the regular step structures and can serve as a spectroscopic tool for the study of vibrational energy states in molecular junctions. We observe a large variation in spectroscopic signatures, as may be expected for the many configurations that the atoms can adopt in the junction. The most frequently encountered structures can be read from a density plot of 1000 such differential conductance traces (Fig. 3), showing VITLS but discarding zero-bias anomalies. The plot shows a high density of points at $35 \mathrm{meV}$ and $48 \mathrm{meV}$ for junctions having a conductance close to $1 \mathrm{G}_{0}$. These vibronic energy values are close to values found by Thijssen et al. in Pt- $\mathrm{D}_{2}-\mathrm{Pt}$ atomic chains [31]. These VITLS signatures support our conclusion that $\mathrm{D}_{2}$ is part of the structure of the ferromagnetic atomic contact.

Additionally, we have taken noise spectra on the ferromagnetic molecular junctions. Due to the discreteness of the electronic charge the electronic current fluctuates around its mean value, which is known as shot noise. For a ballistic conductor shot noise is very sensitive to the transmission probabilities of its electronic energy states. An electron wave traversing the junction will be partially transmitted and partially reflected, if the transmission of the particular channel is not perfect. The partial occupation of forward traveling states and backward reflected states give rise to shot noise. Hence shot noise spectroscopy reveals information on the transmission probabilities and on the numbers of transmission channels involved in electronic transport. For a quantum conductor having $N$ transmission channels with transmission probabilities $\tau_{n}\left(0 \leqslant \tau_{n} \leqslant 1\right)$ the conductance $G$ and shot noise $S_{I}$ can be expressed as [32]

$$
\begin{gathered}
G=\frac{e^{2}}{h} \sum_{n=1}^{N} \tau_{i}, \\
S_{I}=\frac{e^{2}}{h}\left[2 e V \operatorname{coth}\left(\frac{e V}{2 k_{\mathrm{B}} T}\right) \sum_{n=1}^{N} \tau_{n}\left(1-\tau_{n}\right)+4 k_{\mathrm{B}} T \sum_{i=1}^{N} \tau_{n}^{2}\right] .
\end{gathered}
$$



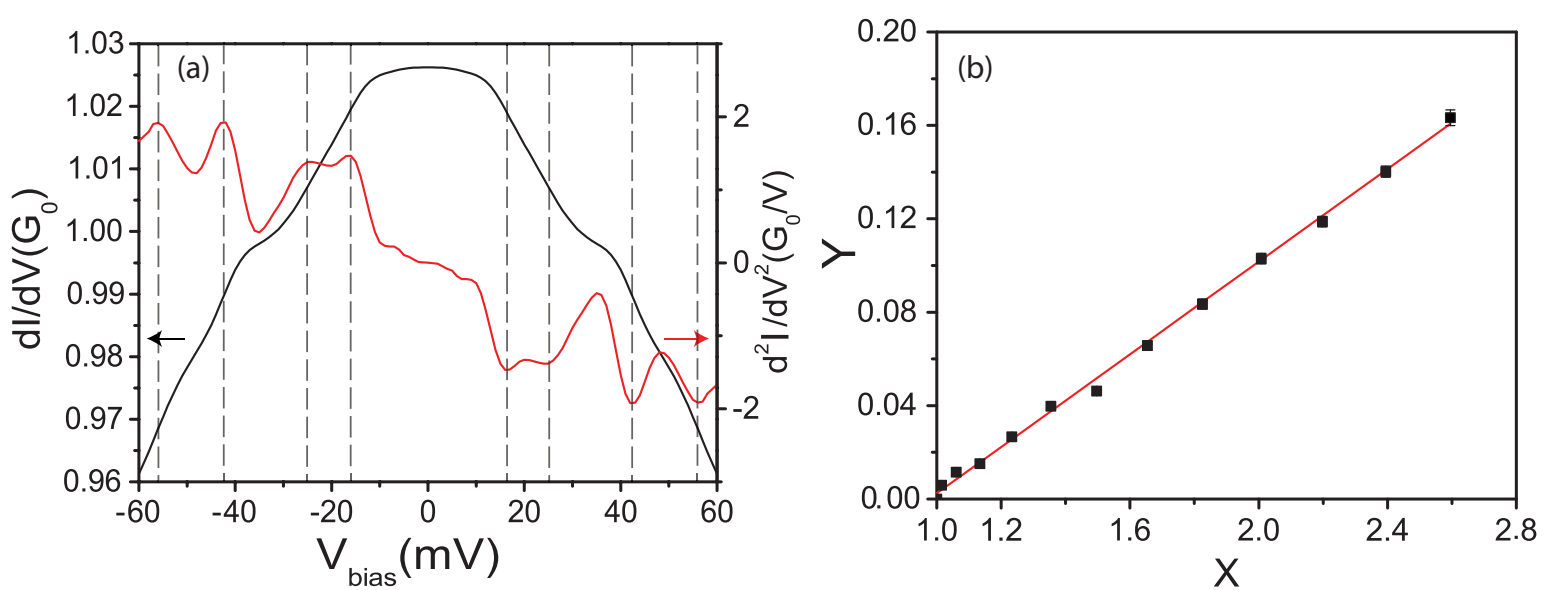

FIG. 4. (Color online) Point contact spectrum (a) for a Ni- $\mathrm{D}_{2}-\mathrm{Ni}$ junction. The inelastic scattering processes are seen in the differential conductance as downward steps, when viewed coming from zero bias. The black curve gives the symmetrized part of the differential conductance; the red curve shows its derivative. Shot noise data are shown in (b) for the same contact as a reduced axis plot. Here $Y=\frac{S_{I}(V)-S_{I}(0)}{S_{I}(0)}$ and $X=\frac{e V}{2 k_{\mathrm{B}} T} \operatorname{coth}\left[\frac{2 e V}{k_{\mathrm{B}} T}\right]$. The Fano factor is obtained from the slope of the linear fit (red line) to the experimental data. The Fano factor for this junction is $0.100 \pm 0.002$.

Here, we have taken the summation over transmission probabilities to include the summation over the spin of the states, i.e., we sum over spin channels. $V$ is the applied bias voltage and $k_{\mathrm{B}} T$ is the thermal energy, with $T$ the bath temperature of the conductor. Equation (2) includes the Johnson-Nyquist thermal noise, which is the equilibrium noise purely defined by the bath temperature $T$. For $e V \gg k_{\mathrm{B}} T$, the nonequilibrium noise dominates and Eq. (2) reduces to $S_{I}=2 e\langle I\rangle F$, where $\langle I\rangle$ is the time averaged current and $F$ is the Fano factor given by

$$
F=\frac{\sum_{n=1}^{N} \tau_{n}\left(1-\tau_{n}\right)}{\sum_{n=1}^{N} \tau_{n}} .
$$

Shot noise spectroscopy is quite sensitive to local configurations of a quantum conductor. For instance, the shot noise in a coherent Landauer conductor will show sub-Poissonian noise, i.e., zero-temperature noise below $2 e\langle I\rangle$, strictly defined by the transmission probabilities of the transmission channels. On the other hand, the presence of localized states in a conductor will give rise to super-Poissionian noise, depending on the ratio of traversal and localized time scales of the current carrying electrons [33]. The latter applies to conductors having twolevel fluctuators [34]. Hence, for the purpose of simplifying the analysis we will restrict noise spectroscopy to junctions having neither VITLS signatures nor Kondo-like zero-bias anomalies in the differential conductance, but that only show molecular signatures as step-down or step-up features.

The experiments started by searching for a stable ferromagnetic molecular junction with chain length of three atoms or longer, which was automated using a Labview controlled dc conductance measurement setup. Once a stable contact was found its differential conductance was measured using a lock-in amplifier. Contacts showing only step features in their differential conductance spectra were selected for the shot noise measurement. Noise spectra were taken at bias settings in steps of $0.25 \mathrm{mV}$ up to $3 \mathrm{mV}$, or even $5 \mathrm{mV}$, depending upon the stability of the junction. The molecular junctions are prone to showing $1 / f$ noise at higher currents, so that we mostly constraint ourselves to low bias noise measurements and the spectral range above the corner frequency of $1 / f$ noise. At the end of each noise measurement series the thermal noise and differential conductance of the contact was measured again. The differential conductance and thermal noise are sensitive to contact configurations and minuscule changes in the configuration can be detected. Noise spectra were only retained for further analysis when no configurational changes were detected.

Figure 4 shows an example of such measurements for a $\mathrm{Ni}-\mathrm{D}_{2}-\mathrm{Ni}$ junction. In order to suppress contributions from conductance fluctuations (due to electron interference as a result of its scattering on defects in the leads [35]) the symmetrized differential conductance is shown, $\frac{1}{2}[G(V)+G(-V)]$. The differential conductance measurement shows vibronic energy features at $16 \mathrm{meV}, 25 \mathrm{meV}$, $43 \mathrm{meV}$, and $56 \mathrm{meV}$. The lowest modes at $16 \mathrm{meV}$ and $25 \mathrm{meV}$ may be associated with local vibrations of the $\mathrm{Ni}$ atomic contact, or possibly $\mathrm{Ni}-\mathrm{D}$ states. However, the highest two can only be associated with light elements, pointing at vibronic states associated with a $\mathrm{D}_{2}$ molecule [21]. Noise spectra were taken for the very same contact at biasing steps of $0.31 \mathrm{mV}$. Figure 4(b) shows a plot of the corresponding noise measurement in reduced units [24]. Here, $Y=\left[S_{I}(V)-S_{I}(0)\right] / S_{I}(0)$ is the noise with the thermal noise removed, and scaled to the thermal noise. The change in bias can be expressed in the scaled variable

$$
X=\frac{e V}{2 k_{\mathrm{B}} T} \operatorname{coth}\left[\frac{2 e V}{k_{\mathrm{B}} T}\right] .
$$

Representing noise data in these reduced units simplifies the expression for noise to a linear relation, from which the Fano factor can be directly obtained as

$$
F=\frac{Y(V)}{(X(V)-1)} .
$$

Thus the Fano factor can be directly obtained from the slope of the reduced axis plot. 


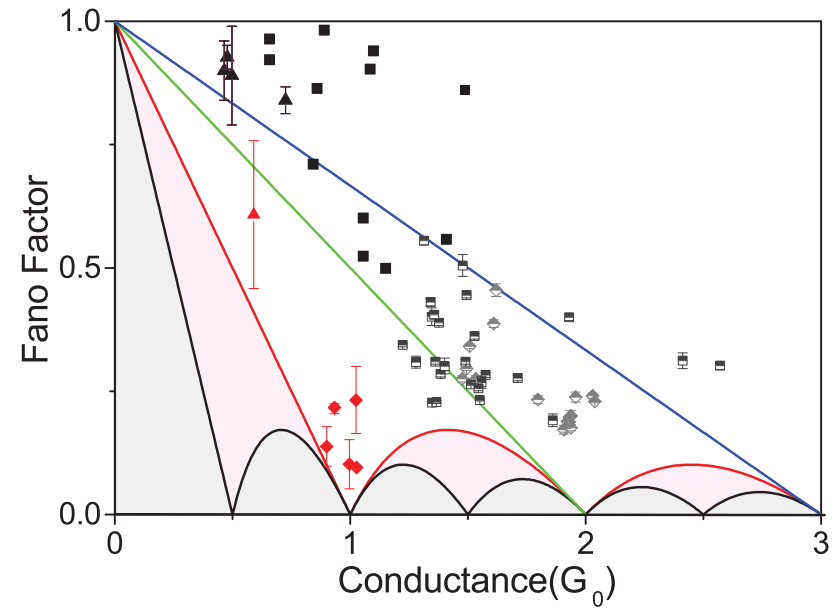

FIG. 5. (Color online) Fano factors measured for ferromagnetic atomic contacts ( $\mathrm{Ni}$ and $\mathrm{Fe}$ ) and ferromagnetic molecular junctions $\left(\mathrm{Ni}-\mathrm{D}_{2}-\mathrm{Ni}\right.$ and $\left.\mathrm{Fe}-\mathrm{D}_{2}-\mathrm{Fe}\right)$. The black curve shows the minimum noise accessible to a spin split Landauer conductor. The red line shows the minimum noise for spin degenerate conductor and light pink region is only accessible for a spin nondegenerate system having highly transmitting spin channels. The green and blue lines show the maximum level of noise for two and three channels, respectively (i.e., four and six spin channels, respectively). The half filled points show the Fano factor measurement for $\mathrm{Ni}$ and $\mathrm{Fe}$ atomic contacts. The ferromagnetic atomic contacts predominantly show the presence of at least three to four transmission spin channels. The fully filled points give Fano factors measured on $\mathrm{Ni}-\mathrm{D}_{2}-\mathrm{Ni}$ and $\mathrm{Fe}-\mathrm{D}_{2}-\mathrm{Fe}$ molecular junctions. The red filled rhombuses are Fano factors measured on $\mathrm{Ni}-\mathrm{D}_{2}-\mathrm{Ni}$ showing molecular step down features in their differential conduction measurements. The red filled triangle shows the Fano factor measured on a $\mathrm{Fe}-\mathrm{D}_{2}-\mathrm{Fe}$ junction showing a step up feature and a small zero bias anomaly in the differential conductance. These junctions show a fully spin degenerate channel and other partially transmitting channels or fully transmitting two spin channels and other two partially transmitting spin channels. The black filled squares and triangles show the Fano factors measured on molecular junctions predominantly showing either Kondo-like zero bias anomalies or VITLS in their differential conductions.

The Fano factor as obtained from the linear fit to the experimental data is $\mathrm{F}=0.100 \pm 0.002$. Figure 5 shows the Fano factors from noise measured on 42 ferromagnetic molecular junctions (36-Ni- $\mathrm{D}_{2}-\mathrm{Ni}$ and $\left.6-\mathrm{Fe}-\mathrm{D}_{2}-\mathrm{Fe}\right)$ and 45 ferromagnetic metal atomic contacts $(20 \mathrm{Fe}$ atomic contacts and $25 \mathrm{Ni}$ atomic contacts). All ferromagnetic molecular junctions showing molecular step features in their differential conductance have lower Fano factors (red filled symbols). In contrast, the presence of either Kondo-like zero-bias anomalies or VITLS in differential conductance leads to higher Fano factors [36]. The presence of localized states in the junctions complicates the story as they tend to give higher noise, or even super-Poissonian noise. Ferromagnetic metal atomic contacts show sub-Poissonian noise indicating the presence of at least three to four transmission spin channels. For the purpose of this paper we will limit our discussion to ferromagnetic molecular junctions showing step features in their differential conductance.

\section{Discussion and conclusion}

The data in Figs. 4 and 5 (red filled symbols only) permit some conclusions regarding the magnetic state of the conductance channels. The transmission probability of channels are calculated using Eqs. (1) and (3). A general procedure for finding the ranges of applicable values for the transmission probabilities can be found in the paper by Vardimon et al. [37]. The small value for the Fano factor implies that all conductance channels either have a small transmission probability, or a transmission close to 1 . For Fig. 4, the combination of measured values for $G / G_{0}=1.03$ and $F=0.100$ are incompatible with less than two spin channels. Expanding the number of channels we find the first solution when assuming three channels. Two of these need to be large, the third small, and a possible solution would be $\tau_{1, \uparrow}=1.00, \tau_{1, \downarrow}=0.91$, and $\left(\tau_{2, \uparrow}=0.15\right.$ or $\left.\tau_{2, \downarrow}=0.15\right)$. This solution is not unique, but represents the essential features of all solutions with three channels and corresponds to weakly spin polarized electron transport. When expanding the analysis to include higher numbers of channels the transmission eigenvalues must lie still closer to either 0 or 1 , with a maximum of two spin channels having a transmission close to 1 . Such solutions are therefore either weakly spin polarized, when the two high transmission channels have opposite spins, or the opposite, strongly spin polarized, when both high-transmission channels have the same spin. The fact that there appears to be one pair of channels close to $\tau=1$ strongly suggests that the $s$-symmetry derived conductance channels dominate the transmission, and that the weakly spin polarized solution is realized. In contrast, the shot noise measurements taken for the pure ferromagnetic atomic contacts show predominantly four, or more, spin channels taking part in the conductance, with no preference for transmission values close to 1 . The wide open transmission channels appear to be characteristic for the $\mathrm{D}_{2}$ modified ferromagnetic atomic contacts, in line with earlier observations for Pt- $\mathrm{D}_{2}-\mathrm{Pt}$ molecular junctions [21].

The presence of $\mathrm{D}_{2}$ apparently filters the channels to produce two high-transmission spin channels. Gracia-Saurez et al. performed $a b$ initio calculations for $\mathrm{Pt}-\mathrm{H}_{2}-\mathrm{Pt}$ and $\mathrm{Pd}-\mathrm{H}_{2}-\mathrm{Pd}$ atomic junctions using the quantum transport code SMEAGOL [38]. They observed that the antibonding state of $\mathrm{H}_{2}$ hybridizes strongly with the $s d_{z^{2}}$ states of the Pt tip atoms, leading to two nearly perfectly transmitting spin channels, while the bonding state is more localized within $\mathrm{H}_{2}$ and does not contribute much towards conduction. Direct calculations for the ferromagnetic molecular junction are still missing, so that one needs to be cautious in comparing our experimental observations with the model calculations of Gracia-Saurez et al.

Our tentative conclusion of weakly spin polarized transport in $\mathrm{Ni}-\mathrm{D}_{2}$ junctions does not imply the absence of local magnetic moments. In our recent study of shot noise related to local magnetic moment formation in Pt atomic chains also found only weakly spin polarized conductance channels. This is still compatible with sizable local magnetic moments, as was demonstrated by means of density-functional calculations [24], because the highly spin polarized states in the Pt chains play a minor role in electron transmission.

Very recently two groups succeeded in obtaining evidence for strong spin polarization from shot noise. Burtzlaff et al. [39] 
used a low-temperature ultrahigh vacuum scanning tunneling microscope for the study of single magnetic adatoms on top of a $\mathrm{Au}(111)$ surface, by means of a Au tip. Touching the adatom ( $\mathrm{Co}$ or $\mathrm{Fe}$ ) with the tip a junction was formed for which conductance and shot noise was recorded. In this case the Au electrodes serve to filter out a single $s$-like channel, but the magnetic atom gives rise to a strongly different transmission between the spin directions, and polarization of the transmission channel of up to $60 \%$ was observed.

Independently, Vardimon et al. [40] performed experiments very similar to the ones presented here, for Ni contacts with the addition of oxygen instead of deuterium. They do not obtain evidence for long atomic chains, but oxygen becomes incorporated into the contacts to form Ni-O-Ni junctions. The evidence from shot noise shows very strong spin polarization of the conductance, even up to $100 \%$. It appears that oxygen plays a very special role, here, with the oxygen $p$ orbitals coupling strongly to the metal $d$ states, thus filtering out the highly spin polarized $d$ orbitals of $\mathrm{Ni}$ for participating in electron transport, rather than the weakly spin polarized $s$ orbitals.

In conclusion, we find that small atoms or molecules added to ferromagnetic junctions may help to limit the number of transmission channels and filter the degree of spin polarization. In addition, in some cases they help to induce spontaneous formation of extended atomic chains forming the junction. Such spin polarized single-channel atomic chains may be a great tool in further experiments on spin polarized quantum transport and spintronics.

\section{ACKNOWLEDGMENTS}

We are grateful to Zhengpen Baardman for assistance in the experiments and to Bert Crama for technical support. This work is part of the research program of the Foundation for Fundamental Research on Matter (FOM), which is financially supported by the Netherlands Organization for Scientific Research (NWO).
[1] A. I. Yanson, G. R. Bollinger, H. E. van den Brom, N. Agräit, and J. M. van Ruitenbeek, Nature (London) 395, 783 (1998).

[2] O. Hideaki, K. Yukihito, and T. Kunio, Nature (London) 395, 780 (1998)

[3] J. C. Cuevas, A. Levy Yeyati, A. Martïn-Rodero, G. R. Bollinger, C. Untiedt, and N. Agräit, Phys. Rev. Lett. 81, 2990 (1998).

[4] G. Rubio-Bollinger, C. de las Heras, E. Bascones, N. Agräit, F. Guinea, and S. Vieira, Phys. Rev. B 67, 121407 (2003).

[5] E. Scheer, P. Konrad, C. Bacca, A. Mayer-Gindner, H. v. Löhneysen, M. Häfner, and J. C. Cuevas, Phys. Rev. B 74, 205430 (2006).

[6] J. Bettini, F. Sato, P. Z. Coura, S. O. Dantas, D. S. Galvào, and D. Ugarte, Nat. Nanotechnol. 1, 182 (2006).

[7] W. H. A. Thijssen, D. Marjenburgh, R. H. Bremmer, and J. M. van Ruitenbeek, Phys. Rev. Lett. 96, 026806 (2006).

[8] S. Csonka, A. Halbritter, and G. Mihály, Phys. Rev. B 73, 075405 (2006).

[9] M. Fischer, A. van Houselt, D. Kockmann, B. Poelsema, and H. J. W. Zandvliet, Phys. Rev. B 76, 245429 (2007).

[10] A. Halbritter, S. Csonka, P. Makk, and G. Mihály, J. Phys.: Conf. Ser. 61, 214 (2007).

[11] R. H. M. Smit, A. I. Mares, M. Häfner, P. Pou, J. C. Cuevas, and J. M. van Ruitenbeek, New J. Phys. 11, 073043 (2009).

[12] P. Makk, Z. Balogh, S. Csonkaa, and A. Halbritter, Nanoscale 4, 4739 (2012).

[13] C. Untiedt, D. M. T. Dekker, D. Djukic, and J. M. van Ruitenbeek, Phys. Rev. B 69, 081401 (2004).

[14] M. R. Calvo, M. J. Caturla, D. Jacob, C. Untiedt, and J. J. Palacios, IEEE Trans. Nanotechnol. 7, 165 (2008).

[15] M. R. Calvo, J. Fernández-Rossier, J. J. Palacios, D. Jacob, D. Natelson, and C. Untiedt, Nature (London) 458, 1150 (2009).

[16] T. Nakazumi and M. Kiguchi, J. Phys. Chem. Lett. 1, 923 (2010).

[17] M. Kiguchi, K. Hashimoto, Y. Ono, T. Taketsugu, and K. Murakoshi, Phys. Rev. B 81, 195401 (2010).
[18] R. H. M. Smit, Y. Noat, C. Untiedt, N. D. Lang, M. C. van Hemert, and J. M. van Ruitenbeek, Nature (London) 419, 906 (2002).

[19] M. Kumar, Ph.D. thesis, Leiden University, 2012.

[20] H. E. van den Brom and J. M. van Ruitenbeek, Phys. Rev. Lett. 82, 1526 (1999).

[21] D. Djukic and J. M. van Ruitenbeek, Nano Lett. 6, 789 (2006).

[22] A. Kumar, L. Saminadayar, D. C. Glattli, Y. Jin, and B. Etienne, Phys. Rev. Lett. 76, 2778 (1996).

[23] C. Untiedt, A. I. Yanson, R. Grande, G. Rubio-Bollinger, N. Agräit, S. Vieira, and J. M. van Ruitenbeek, Phys. Rev. B 66, 085418 (2002).

[24] M. Kumar, R. Avriller, A. L. Yeyati, and J. M. van Ruitenbeek, Phys. Rev. Lett. 108, 146602 (2012).

[25] S. R. Bahn, N. Lopez, J. K. Nørskov, and K. W. Jacobsen, Phys. Rev. B 66, 081405 (2002).

[26] M. Galperin, M. A. Ratner, and A. Nitzan, J. Chem. Phys. 121, 11965 (2004).

[27] J. K. Viljas, J. C. Cuevas, F. Pauly, and M. Häfner, Phys. Rev. B 72, 245415 (2005).

[28] L. de la Vega, A. Martín-Rodero, N. Agraït, and A. Levy Yeyati, Phys. Rev. B 73, 075428 (2006).

[29] O. Tal, M. Krieger, B. Leerink, and J. M. van Ruitenbeek, Phys. Rev. Lett. 100, 196804 (2008).

[30] See Supplemental Material by M. Kumar, K. V. S. Kumar, and J. M. van Ruitenbeek at http://link.aps.org/supplemental/ 10.1103/PhysRevB.91.245404 for molecule-assisted ferromagnetic atomic chain formation.

[31] W. H. A. Thijssen, D. Djukic, A. F. Otte, R. H. Bremmer, and J. M. van Ruitenbeek, Phys. Rev. Lett. 97, 226806 (2006).

[32] Y. Blanter and M. Büttiker, Phys. Rep. 336, 1 (2000).

[33] W. Belzig, Phys. Rev. B 71, 161301 (2005).

[34] A. Carmi and Y. Oreg, Phys. Rev. B 85, 045325 (2012).

[35] B. Ludoph, M. H. Devoret, D. Esteve, C. Urbina, and J. M. van Ruitenbeek, Phys. Rev. Lett. 82, 1530 (1999). 
[36] Data shown here only for contacts having $0<F<1$. The majority of contacts having zero bias Kondo-like anomalies or VITLS in the differential conductance have super-Poissonian noise.

[37] R. Vardimon, M. Klionsky, and O. Tal, Phys. Rev. B 88, 161404 (2013).
[38] V. M. García-Suárez, A. R. Rocha, S. W. Bailey, C. J. Lambert, S. Sanvito, and J. Ferrer, Phys. Rev. B 72, 045437 (2005).

[39] A. Burtzlaff, A. Weismann, M. Brandbyge, and R. Berndt, Phys. Rev. Lett. 114, 016602 (2015).

[40] R. Vardimon, M. Klionsky, and O. Tal (unpublished). 